\title{
Developmental regulation of Dirofilaria immitis microfilariae and evaluation of ecdysone signaling pathway transcript level using droplet digital PCR
}

\author{
Tsai-Chi Shang Kuan ${ }^{*}$ and Roger K. Prichard ${ }^{*}$
}

\begin{abstract}
Background: Current measures for the prevention of dirofilariasis, caused by the dog heartworm, Dirofilaria immitis, rely on macrocyclic lactones, but evidence of drug-resistant isolates has called for alternative approaches to disease intervention. As microfilariae are known to be in a state of developmental arrest in their mammalian host and then undergo two molts once inside the arthropod, the aim of this study was to look at the developmental regulation of D. immitis microfilariae that occurs in their arthropod host using in vitro approaches and to investigate the role of the ecdysone signaling system in this development regulation.
\end{abstract}

Methods: Dirofilaria immitis microfilariae extracted from dog blood were incubated under various culture conditions to identify those most suitable for in vitro culture and development of the microfilariae, and to determine the effects of fetal bovine serum (FBS), mosquito cells, and ecdysteroid on the development of the microfilariae. Transcript levels of the ecdysone signaling pathway components were measured with droplet digital PCR (ddPCR).

Results: In vitro conditions that best promote early development of D. immitis microfilariae to the "late sausage stage" have been identified, although shedding of the cuticle was not observed. FBS had inhibitory effects on the development and motility of the microfilariae, but media conditioned with Anopheles gambiae cells were favorable to microfilarial growth. The transcript level study using ddPCR also showed that ecdysone signaling system components were upregulated in developing microfilariae and that 20-hydroxyecdysone increased the proportion of larvae developing to the sausage and late sausage stages in vitro.

Conclusions: The arthropod host environment provides cues required for the rapid development of $D$. immitis microfilariae, and the ecdysone signaling system may play an important role in filarial nematode developmental transitions. This study contributes to a better understanding of the developmental process of $D$. immitis microfilariae.

Keywords: Dirofilaria immitis, Microfilaria, Anopheles gambiae, Ecdysone signaling pathway, 20-Hydroxyecdysone

\section{Background}

Dirofilaria immitis, also called the dog heartworm, is the causative agent of cardiovascular dirofilariasis, a serious and potentially fatal disease in pets that is spread

\footnotetext{
*Correspondence: tsai-chi.shangkuan@mail.mcgill.ca; roger.prichard@mcgill.ca Institute of Parasitology, McGill University, Sainte-Anne-de-Bellevue, Montreal H9X3V9, Canada
}

by mosquitoes. All canids, regardless of age or sex, are highly susceptible to heartworm infections [1]. According to the 2019 American Heartworm Society (AHS) incidence survey, the total number of cases of dogs with heartworm disease reported in the USA has risen by about $12 \%$ since the organization's first survey 18 years ago. Prophylaxis relies heavily on macrocyclic lactones 
(ML), which mainly target the third- and fourth-stage larvae, but long-term use of ML has led to the development of drug-resistant isolates, challenging current therapeutic control [2-8]. Studying the regulation of other developmental stages of the nematode thus provides an attractive approach to identifying alternative therapeutic targets.

Dirofilaria immitis requires an arthropod and a mammalian host to complete its life cycle. Inside the mammalian host, adult female worms residing in the pulmonary vasculature can release high numbers of microfilariae into the circulation after mating with the male worms [9]. These microfilariae remain in a state of developmental arrest in the bloodstream for up to 2.5 years until an arthropod vector ingests them $[10,11]$. A wide range of mosquito species can be exploited by the parasite [12, 13]. Upon being taken up by a mosquito, microfilariae will migrate from the midgut to the Malpighian tubules, where they quickly undergo developmental transitions known as molts, or the shedding of the old cuticle. At temperatures between $23{ }^{\circ} \mathrm{C}$ and $27{ }^{\circ} \mathrm{C}$, the first molt, in the mosquito, usually takes place between 8 and 10 days post-infection and causes the stoma to open to the exterior. The second molt to the third-stage (L3) larvae takes place around 2-3 days after the first molt [14-17]; this molt removes the anal plug $[18,19]$. Our current knowledge of these stages indicates that the developmental cues that trigger molting are closely tied to the transition to a new host environment. Inhibition of the molting process would thus arrest the life cycle and prevent the pathology of dirofilariasis.

Similar to nematodes, arthropods are ecdysozoans that develop through a series of periodic larval stage molts before reaching adulthood [20]. Key pathways that regulate the growth of insects have been well-characterized: cell and tissue growth rates are known to be controlled by the insulin/insulin-like growth factor signaling pathway, whereas developmental transitions and metamorphosis are under the control of the ecdysone signaling pathway [21]. Ecdysteroids are sterol derivatives that bear similar structural features and are synthesized in arthropods from dietary cholesterol upon metabolism by cytochrome P450 enzymes. 20-Hydroxyecdysone (20E), the active ecdysteroid hormone that regulates the physiological and behavioral changes during insect molting [22], is converted from ecdysone by the ecdysone 20-monooxygenase in peripheral tissues, such as the fat body, ovaries, midgut, and Malpighian tubules [23-26]. The hormonal action of $20 \mathrm{E}$ is exerted through binding to the ecdysone receptor (EcR), a heterodimeric nuclear receptor (NR) composed of EcR and the ultraspiracle protein (USP), the insect orthologue of the mammalian retinoid X receptor protein (RXR) [27-29]. Upon activation, EcR/USP directly activate a cascade of gene expression by recognizing the ecdysone-response element (EcRE) $5^{\prime}-(\mathrm{A} / \mathrm{G}) \mathrm{G}(\mathrm{G} / \mathrm{T})$ T C A N T G A (C/A) $\mathrm{C}(\mathrm{C} / \mathrm{T})-3^{\prime}$ in the promoter region of its target genes $[30,31]$. According to the Ashburner model [32, 33], this leads to the expression of a set of early regulatory genes (at least six in Drosophila melanogaster, including E74, E75, E78, HR3, HR4, and $\beta F t z-F 1$ ) [34], but represses the expression of the late regulatory genes. The proteins of the early genes then repress their own promoters while inducing the expression of the late genes, whose protein products play a more direct role in controlling molting and metamorphosis.

As in insects, a possible candidate pathway that controls molting in filarial nematodes is the ecdysone signaling pathway. Both free and conjugated ecdysteroids have been detected in the larval and adult stages of several species of filarial nematodes, including D. immitis and Onchocerca volvulus $[35,36]$. Elements of the ecdysone signaling pathway and the NR that function downstream of EcR and USP have also been identified in D. immitis [37-40]. In addition, evidence for a functional ecdysone signaling system in filarial nematodes was also demonstrated in Brugia malayi in a study by Tzertzinis et al. [41]. When exogenously applied, ecdysteroids have been shown to exert biological effects on the molting of third-stage and fourth-stage nematodes. For example, low concentrations of $20 \mathrm{E}$ were found to stimulate the molting of D. immitis [42] and Ascaris suum third-stage larvae [43], as well as Heligmosomoides polygyrus fourthstage larvae [44]. In addition, non-steroidal ecdysteroid agonists (RH compounds) were also found to induce the molting of third-stage larvae of D. immitis [42]. Although the role that the ecdysone signaling pathway plays in the developmental transition of third-stage and fourth-stage larvae is evident, the role of ecdysteroids in microfilarial development is largely unknown.

This study attempts to establish an in vitro culture condition similar to the mosquito host environment to determine factors that stimulate the development of D. immitis microfilariae and to identify the role of the ecdysone signaling system during the developmental process. This work advances understanding of $D$. immitis microfilaria in vitro development and offers insights to novel targets for drug development.

\section{Methods}

\section{In vitro culture of $D$. immitis microfilariae}

Microfilariae of D. immitis (2005 Missouri strain) were provided by FR3 Molecular Resources through the BEI Resources Repository [45]. Blood samples containing microfilariae were collected from infected dogs and shipped overnight to McGill University. Upon arrival, the blood samples were diluted with $\mathrm{NaHCO}_{3}$ 
(Sigma-Aldrich, St. Louis, MO, USA) and filtered through $3.0-\mu \mathrm{m}$ polycarbonate membrane filters as previously described [46]. After several washes in phosphate buffered saline (PBS), microfilariae were plated in a 24-well plate at a density of 500 microfilariae per well in culture media supplemented with $100 \mathrm{U} / \mathrm{ml}$ penicillin (Gibco ${ }^{\mathrm{TM}}$; Thermo Fisher Scientific, Waltham, MA, USA ), $100 \mu \mathrm{g} / \mathrm{ml}$ streptomycin $\left(\right.$ Gibco $^{\mathrm{TM}}$ ), $0.25 \mu \mathrm{g} / \mathrm{ml}$ amphotericin B $\left(\right.$ Gibco $\left.^{\mathrm{TM}}\right)$, and $0.01 \mathrm{mg} / \mathrm{ml}$ gentamicin (Sigma-Aldrich). The media tested included RPMI-1640 with L-glutamine $\left(\right.$ Gibco $\left.^{\mathrm{TM}}\right)$, Dulbecco's modified Eagle's medium (DMEM) with high glucose and L-glutamine $\left(\right.$ Gibco $\left.^{\mathrm{TM}}\right)$ levels, Ham's F-12 (Nutrient Mix) with L-glutamine $\left(\right.$ Gibco $\left.^{\mathrm{TM}}\right)$, DMEM and Ham's F-12 mixed at a 1:1 ratio, and Schneider's insect medium with L-glutamine (Sigma-Aldrich). The parasites were incubated at $26^{\circ} \mathrm{C}$ in $5 \% \mathrm{CO}_{2}$ for 22 days, with/without the presence of Anopheles gambiae cells or fetal bovine serum (FBS). To test the effects of serum, $10 \%(\mathrm{v} / \mathrm{v})$ heat-inactivated FBS $\left(\right.$ Gibco $\left.^{\mathrm{TM}}\right)$ was added to each well.

To assess the effects of ecdysteroid on the development of microfilariae in vitro, microfilariae were incubated in DMEM and Ham's F-12 mixed at a 1:1 ratio without $A n$. gambiae cells or conditioning with these cells. 20E (Cayman Chemical Co., Ann Arbor, MI, USA) dissolved in DMSO was added to the incubation at a final concentration of $5 \mu \mathrm{M}, 0.05 \%(\mathrm{v} / \mathrm{v})$ DMSO. To increase the possible uptake of 20E to the D. immitis larvae, $0.004 \%(\mathrm{v} / \mathrm{v})$ (2-hydroxypropyl)- $\beta$-cyclodextrin (Sigma-Aldrich) was included in the control and $20 \mathrm{E}$ incubations.

\section{Anopheles gambiae cell culture}

Anopheles gambiae cell line 4a-3B (provided by Dr. George K. Christophides) was maintained at $26^{\circ} \mathrm{C}$ in $5 \%$ $\mathrm{CO}_{2}$ in Schneider's insect medium supplemented with $10 \%(\mathrm{v} / \mathrm{v})$ heat-inactivated FBS, $100 \mathrm{U} / \mathrm{ml}$ penicillin, and $100 \mu \mathrm{g} / \mathrm{ml}$ streptomycin. For co-culture with $D$. immitis microfilariae, An. gambiae cells were seeded at a density of $5 \times 10^{4}$ cells $/ \mathrm{cm}^{2} 48 \mathrm{~h}$ prior to co-culturing and washed with PBS $24 \mathrm{~h}$ later. The cells were then incubated in the culture medium intended for culturing the parasite for the remaining $24 \mathrm{~h}$ prior to introducing the microfilariae. Cells were re-seeded every 3 days. To condition media with An. gambiae cells, cells were seeded at the same density as mentioned above, and after $24 \mathrm{~h}$ they were washed with PBS and incubated in the culture medium for another $24 \mathrm{~h}$. Only the cell culture medium was collected and used for $D$. immitis culture.

\section{Development of $D$. immitis microfilariae}

Microfilariae were observed every $24 \mathrm{~h}$ during the 22-day incubation, and culture media were renewed every day with the respective fresh complete medium. Body dimensions of the parasites were measured and analyzed with the Nikon NIS-Elements Advanced Research image analysis software (Nikon Corp., Tokyo, Japan). Morphological changes in the parasites were noted, and the motility of the parasite was measured as any movement within a 30-s timeframe. The percentage of each stage of development was calculated as: (mean of stage-specific parasite from a triplicate or quadruplicate assay/total number of microfilariae) $\times 100$.

The IncuCyte live-cell imaging and analysis platform was used for the live worm analysis. Live microfilariae were cultured in 24-well cell culture plates at $26^{\circ} \mathrm{C}$, $5 \% \mathrm{CO}_{2}$ and imaged with the IncuCyte ZOOM system (Essen BioScience, Royston, Hertfordshire, UK) at $20 \times$ magnification.

\section{Scanning electron microscopy}

Microfilariae were pre-chilled at $4{ }^{\circ} \mathrm{C}$ for $30 \mathrm{~min}$ and placed in fixative of $2.5 \%$ glutaraldehyde and $1.5 \%$ formaldehyde in PBS for $2 \mathrm{~h}$. The samples were then washed with PBS $(3 \times 10 \mathrm{~min})$ and dehydrated in an ethanol series of $30 \%$ ( $1 \times 10 \mathrm{~min}), 50 \%$ ( $1 \times 10 \mathrm{~min}), 70 \%(1 \times 10 \mathrm{~min}), 80 \%$ $(1 \times 10 \mathrm{~min}), 90 \%(1 \times 10 \mathrm{~min})$, and $100 \%(3 \times 30 \mathrm{~min})$. Dehydrated samples were dried in a critical point dryer by $\mathrm{CO}_{2}$ treatment (Leica EM CPD300; Leica Microsystems, Wetzlar, Germany) or chemically dried with hexamethyldisilazane (HMDS) (Sigma-Aldrich) $(3 \times 15 \mathrm{~min})$. Dried samples were sputter-coated with $4 \mathrm{~nm}$ of gold and palladium using the Leica EM ACE200 coater (Leica Microsystems) and mounted onto aluminum stubs for scanning electron microscopy (SEM) observation (Hitachi TM-1000 EM microscope; Hitachi Ltd., Tokyo, Japan).

\section{Quantification of transcript level}

Adult $D$. immitis samples (Kentucky strain, ML-susceptible strain) were provided by Zoetis Inc. (Parsippany-Troy Hills, NJ, USA) for the transcript-level study. The adult worms were obtained through necropsy of experimentally-infected dogs and flash-frozen upon collection.

Total RNA from $6-8 \times 10^{4} \mathrm{D}$. immitis microfilariae (Missouri strain) and individual male or female adult worms (Kentucky strain) was isolated with TRIzol ${ }^{\mathrm{TM}}$ reagent (Ambion, Invitrogen, Thermo Fisher Scientific), following the manufacturer's protocol. Homogenization was performed by crushing the parasite using plastic pestles and 425- to 600- $\mu \mathrm{m}$ glass beads (Sigma-Aldrich). Extracted RNA was treated with DNase using an Invitrogen DNA-free kit (Thermo Fisher Scientific). RNA concentration was assessed with a NanoDrop One ${ }^{\mathrm{C}}$ spectrophotometer (Thermo Fisher Scientific), and the quality determined by running an aliquot of the RNA sample on a native agarose gel. cDNA was obtained by reverse 
transcription using the SuperScript III first-strand synthesis system (Thermo Fisher Scientific) with oligo $(\mathrm{dT})_{20}$ primers.

Detection of target genes and validation of primers (Additional file 1: Table S1) were performed with PCR and quantitative (q) PCR using Taq DNA polymerase (Invitrogen, Thermo Fisher Scientific) and $2 \times$ SYBR Select Master Mix (Applied Biosystems, Foster City, CA, USA), respectively. The absolute quantification of target nucleic acids present in the sample was determined by droplet digital PCR (ddPCR). In brief, the ddPCR mix comprised 2× QX200 ddPCR EvaGreen Supermix (BioRad, Hercules, CA, USA), $250 \mathrm{nM}$ final concentration of each forward and reverse primer, cDNA sample, and nuclease-free water. Droplet generation oil (Bio-Rad) was added to the ddPCR mix and the mixture then partitioned into 20,000 nanoliter-sized droplets by a QX200 ${ }^{\mathrm{TM}}$ Droplet Generator (Bio-Rad). The droplets were then subjected to standard PCR amplification and read with a QX200 ${ }^{\mathrm{TM}}$ Droplet Reader (Bio-Rad) to determine the target concentration using Poisson's statistics [47]. Results were analyzed by normalizing the copy number of the gene of interest against the geometric mean of the copy numbers of three reference genes. The fold change in transcript level was then calculated by comparing the normalized value to the baseline (day 1) transcript level.

\section{Statistical analysis}

All experiments were performed with at least three individual replicates. Data presented for the in vitro culture of $D$. immitis are shown as mean data \pm standard deviation (SD). The effects of Anopheles gambiae cells on the proportion of the microfilariae developing to a later stage in vitro and on the proportion of each stage that were seen to be motile were analyzed by Kruskal-Wallis H-test with Dunn's multiple comparisons test. The effects of $20 \mathrm{E}$ on the proportion of the microfilariae developing to a later stage in vitro and the proportion of each stage that were seen to be motile were analyzed by Mann-Whitney U-test, two-tailed $P$ value, and 95\% confidence interval. The transcript levels of the ecdysone signaling system genes were analyzed by unpaired t-test with Welch's correction, two-tailed $P$ value, and 95\% confidence interval. All analyses was performed using Prism 6.0c (Graph Pad Software, Inc., San Diego, CA, USA).

\section{Results}

Microfilariae develop into pre-sausage, sausage, and late sausage stages

As $D$. immitis microfilariae develop, they progress into morphologically distinct forms that can be categorized as microfilaria, pre-sausage, sausage, or late sausage stage (Fig. 1a-d). When first extracted from dog blood samples, parasites were in the microfilaria stage and appeared elongated and slender, with vigorous serpentine movements (Additional file 2: Video S1). When microfilariae were incubated in vitro, the body of the parasite shortened and the posterior region began to enlarge after several days, making the tail more distinct; this stage was classified as the pre-sausage stage. Compared to the microfilaria stage, the pre-sausage stage was more granular in appearance, and parasite movement was slowed (Additional file 3: Video S2). Over the next few days, the pre-sausage stage larvae grew shorter in length, resulting in a stumpy appearance with a fine tail at the tip, approaching the sausage stage. The sausage stage was more basophilic than the microfilaria, with heavy internal granulation, and movement was sluggish (Additional file 4: Video S3). At the late sausage stage, movement of the parasite continued to decline (Additional file 5: Video S4), and at the same time the anterior region of the sausage stage larvae started to enlarge while the body became more elongated. The length and width of the parasites were measured to determine the mean body dimensions for the different stages (Fig. 1e).

\section{Pre-sausage, sausage, and late sausage stage parasites are first-stage larvae}

To further study the morphological differences between the microfilaria, pre-sausage, sausage, and late sausage stage parasites and to observe possible signs of molting, SEM analysis and live-cell analysis were performed. Numerous transverse grooves in the cuticle were evident in all developmental stages, giving the parasite a striated appearance (Fig. $2 \mathrm{a}-\mathrm{d}$ ). The grooves seemed to become more noticeable as the parasite became more developed. No visible difference was seen in the stoma region among the four stages; all of the parasites had a circular tissue located at the tip of the head and layers of folded tissue that surrounded it. Results of the live-cell analysis with the IncuCyte ZOOM system also showed that development to the late sausage stage was not accompanied by any shedding of the cuticle (Additional file 6: Video S5), although separation of the outer cuticle could be seen.

\section{DMEM/Ham's F-12 medium provides a relatively favorable condition for the in vitro cultivation of $D$. immitis microfilariae}

To determine suitable conditions for the in vitro cultivation of $D$. immitis microfilariae, extracted microfilariae were incubated in various commercially available culture media at $26{ }^{\circ} \mathrm{C}$ for 22 days. Results show that both RPMI 1640 and Schneider's insect medium were poor at sustaining the development and motility of 
the microfilariae (Fig. 3a, b). DMEM medium maintained microfilariae motility but was unable to initiate development to the pre-sausage stage (Fig. 3c), while Ham's F-12 was beneficial to their development. Reduced motility was associated with this development (Fig. 3d). When mixed at a 1:1 ratio, DMEM/Ham's F-12 provided a favorable condition for the microfilariae and supported larvae development to the pre-sausage and sausage stage (Fig. 3e).

When $10 \%$ heat-inactivated FBS was added to the microfilaria culture, both development and motility of the parasite were drastically reduced, and microfilariae appeared to be more lethargic and degenerate, with little development to the pre-sausage stage observed in most groups (Fig. 3a, b, d, e). An exception was seen in those that were incubated in DMEM and 10\% FBS (Fig. 3c); although development was initiated in this group and some pre-sausages developed into sausages, most of the parasites appeared to be degenerated and motility was greatly reduced compared to the group without $10 \%$ FBS.

\section{Anopheles gambiae cells and cell-secreted factors promote D. immitis microfilariae development}

Anopheles gambiae cells were introduced into the culture of microfilariae incubated in DMEM/Ham's F-12 for 22 days to test the effect of insect cells on the development of the microfilariae. Consistent with results shown in Fig. 3, larvae incubated in DMEM/F-12 in the absence of mosquito cells developed into the pre-sausage stage at around day 4, but few developed further into the sausage stage throughout the 22-day incubation period. The presence of An. gambiae cells, however, initiated faster development, and significantly higher levels of sausage stages were observed as early as day 7 (Fig. 4a, b); also, some parasites were able to develop into the late sausage stage.

When medium conditioned with An. gambiae cells was used instead of having physical cells in the culture, a similar effect in the development of microfilariae was also seen. In fact, there was a significant decrease in the percentage of microfilaria and an increase in the number of pre-sausage stage larvae during early incubation. In addition, more sausage stage larvae were observed when the cell-conditioned medium was used compared to when microfilariae were in physical contact with the insect cells (Fig. 4c, d).

While motility always decreased as the microfilariae developed to the pre-sausage and later stages, the presence of cells and cell-conditioned media seemed to better maintain parasite activity, whereas in the absence of cells, motility dropped at a faster rate and the parasites

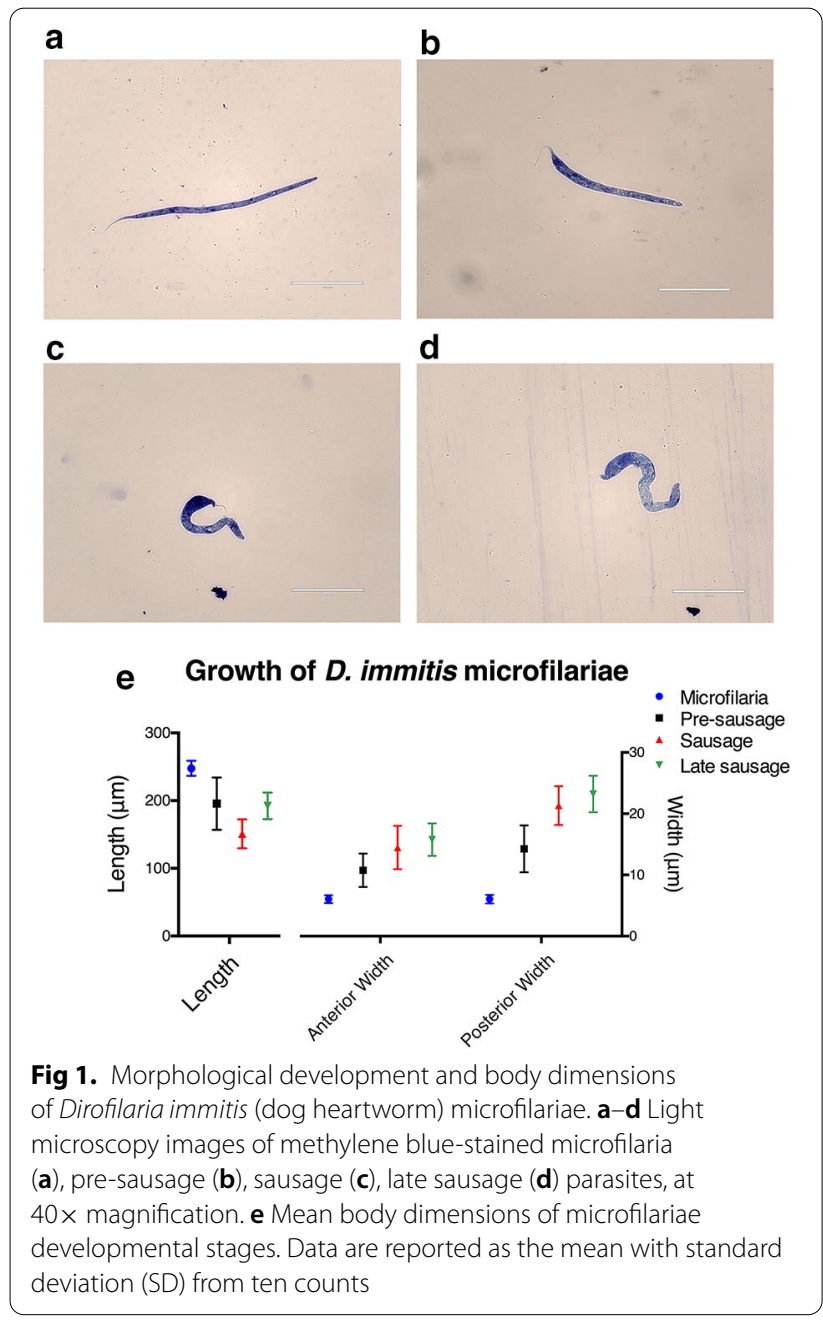

appeared more sluggish. The presence of cells in the culture also appeared to slow the drop in motility compared to parasites cultured in cell-conditioned media, although the motility results were not significantly different (Fig. 4e).

\section{$E c R, r \times r-1$ and downstream early regulatory genes are upregulated in developing microfilariae}

As steroid-nuclear hormone receptor signaling plays an important role in ecdysozoan development [48], microfilariae were collected every 3 days, and the transcript levels of the Dim-EcR, Dim-rxr-1, and downstream early regulatory genes were measured to determine the transcriptional change in the ecdysteroid signaling pathway during microfilarial developmental changes. Dim-nhr-7 and Dim-nhr-6, orthologues of E78 and E75, respectively, are both downstream early regulatory genes of the ecdysone receptor. The putative EcRE was found in 

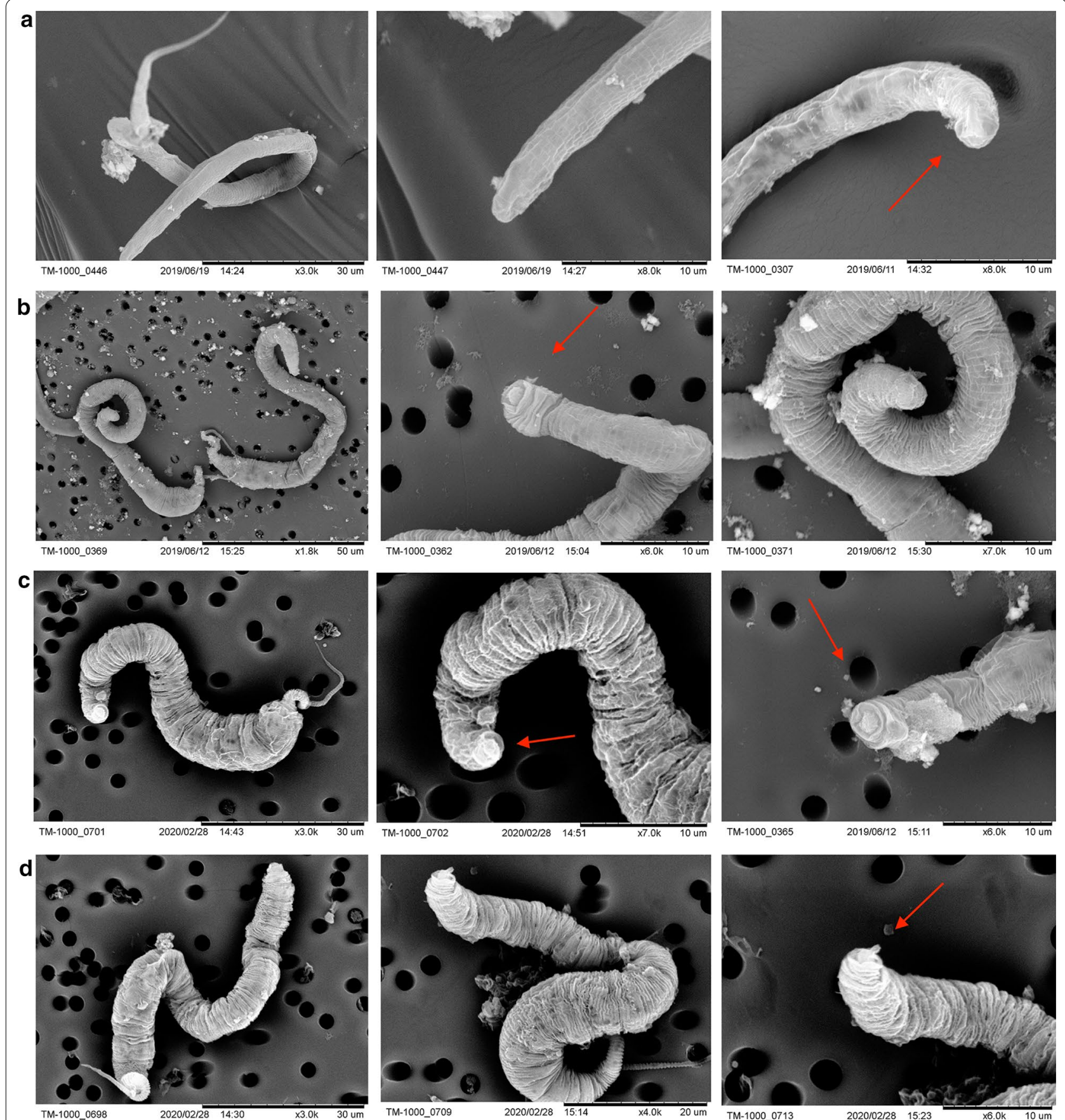

Fig 2. Scanning electron miscropy analysis of D. immitis microfilaria, pre-sausage, sausage, and late sausage stages. a Microfilaria mounted on a double-sided tape showing whole parasite (left), enlarged head region (middle), ventral anterior view (right). b Pre-sausage stage larvae mounted on a 3.0- $\mathrm{mm}$ polycarbonate membrane filter showing whole parasites (left), enlarged head region (middle and right). c Sausage stage larvae mounted on a 3.0- $\mu \mathrm{m}$ polycarbonate membrane filter showing whole parasite (left) and enlarged head region (middle and right). $\mathbf{d}$ Late sausage stage larvae mounted on a 3.0- $\mu$ m polycarbonate membrane filter, showing whole parasite (left and middle) and enlarged region (right). Red arrows show where the stoma opening would be 
the upstream promoter regions of both Dim-nhr-7 and Dim-nhr-6, indicating that Dim-nhr-7 and Dim-nhr-6 can potentially be regulated by EcR directly (Fig. 5). All primer pairs (Additional file 1: Table S1) were optimized for specificity and efficiency, and the quantification cycle (Cq) values from qPCR were used to estimate template concentrations for use on the ddPCR. After normalizing the genes of interest to the three reference genes
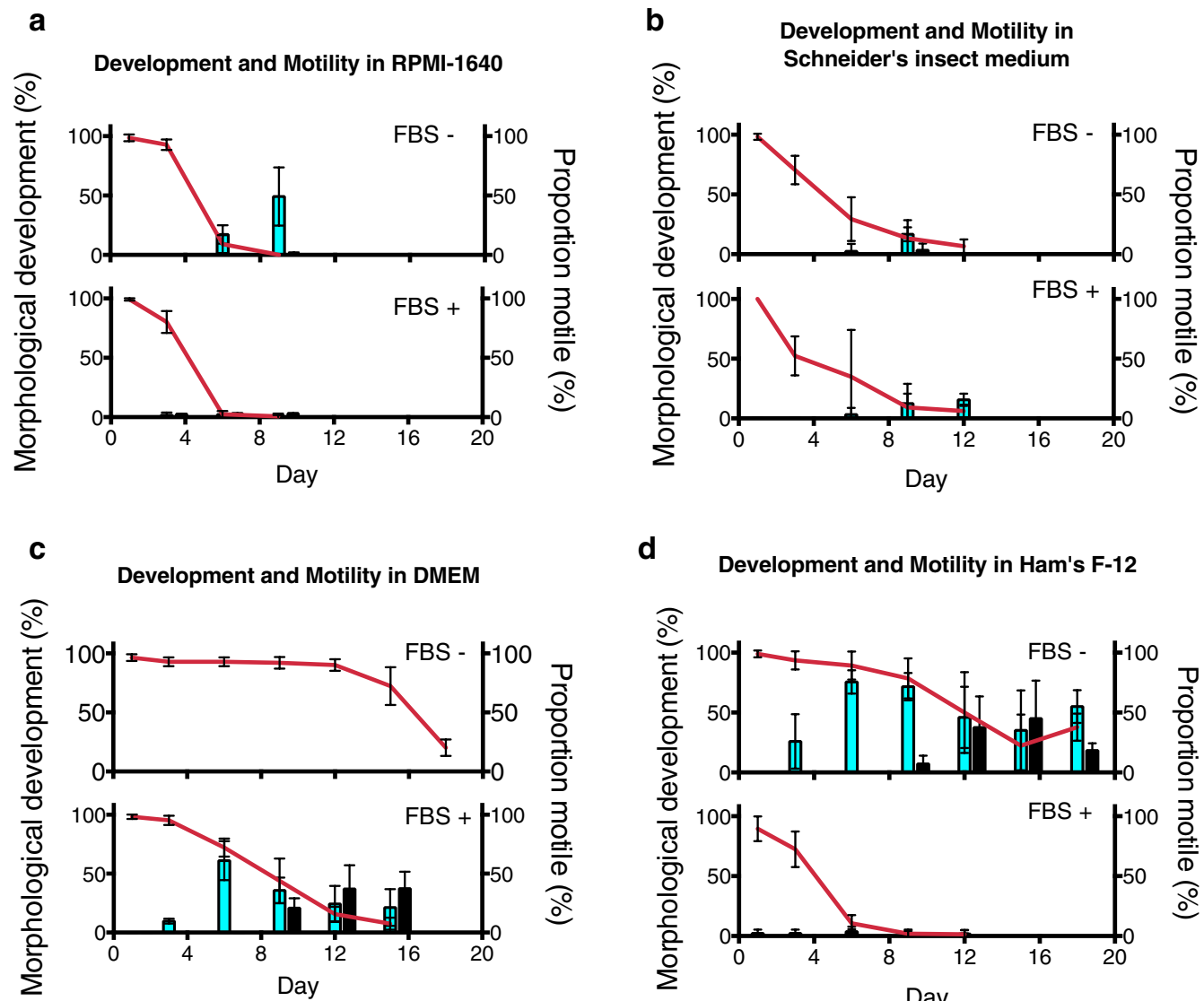

d

Development and Motility in Ham's F-12
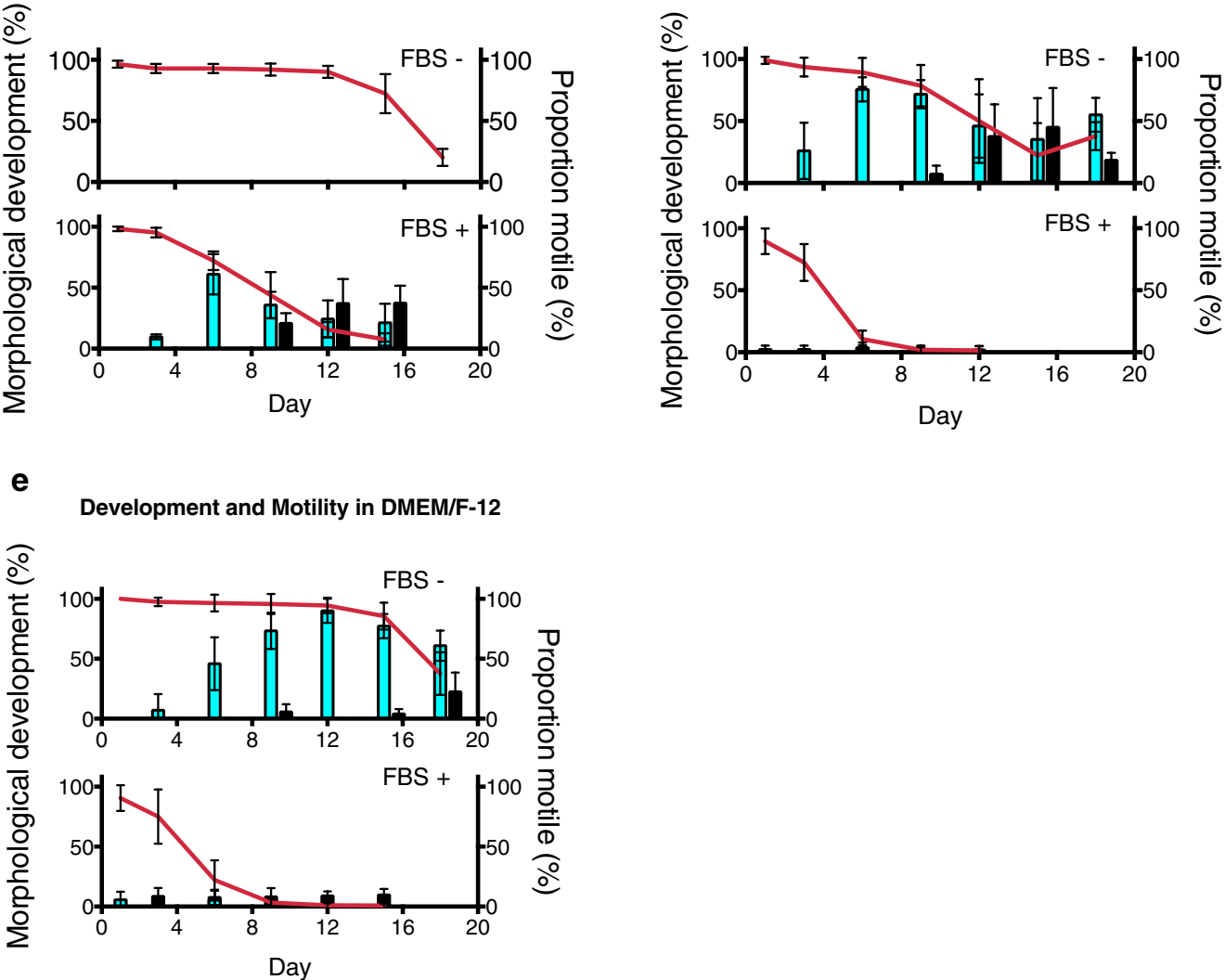

Fig 3. Development of $D$. immitis microfilariae and proportion of motile larvae in commercially available media and fetal bovine serum (FBS). a RPMI-1640 medium, b Schneider's insect medium, c DMEM, d Ham's F-12, e DMEM/Ham's F-12. All data are reported as mean percentages, with error bars corresponding to the SD, from at least four individual replicates 
Dim-GAPDH, Dim-Actin, and Dim- $\beta$-tubulin, Dim-EcR, Dim-rxr-1, Dim-nhr-7, and Dim-nhr-6 were all upregulated to different magnitudes in developing first-stage (L1) larvae, as shown in Fig. 6a-d. In both no-cell and An. gambiae cell-conditioned media groups, transcript levels of all four genes consistently increased during the first few days of development. However, in no-cell groups, the transcript levels of all four genes incurred a drop at around day 13-16, following which an increase was seen again on day 19. In groups cultured in the cellconditioned medium, a gradual increase throughout the 22-day incubation period was seen for $D i m-E c R$, Dim-rxr-1, and Dim-nhr-6, with a sharp rise on day 22 . In contrast, the transcript level of Dim-nhr-7 in the cellconditioned medium group remained relatively constant during the early incubation period, but a sharp rise could be seen on day 22 .

Overall, developing larvae in cell-conditioned medium groups expressed higher transcript levels than those that were not incubated in cell medium, with the exception being the transcript level of Dim-nhr-7, for which the nocell group has a higher transcription level, but the transcript level of the cell-conditioned medium group quickly rose higher on day 22. In addition, the overall transcript level of Dim-nhr-6 in developing microfilariae was significantly higher than those of the other three genes. Transcript levels of female and male adults were also included as a comparison, and the results showed that the transcript level of the ecdysteroid signaling system components was higher in females than in males for all four genes.

\section{0-Hydroxyecdysone promotes development to sausage and late sausage stage larvae}

In view of the upregulation of genes involved in the ecdysone regulatory development pathway, the effects of $20 \mathrm{E}$ were investigated on microfilariae cultured in vitro, without insect cells or in insect cell-conditioned medium. When microfilariae were treated with $5 \mu \mathrm{M} 20 \mathrm{E}$ every day, with (2-hydroxypropyl)- $\beta$-cyclodextrin (HP- $\beta-\mathrm{CD})$ as the carrier of the hydrophobic compound, there was a significantly higher level of total pre-sausage stage larvae at day 19 and a significantly higher level of sausage stage larvae in the culture at the end of the 22-day incubation, compared to the DMSO + HP- $\beta-C D$ control group. With the increase in development to the sausage stage larvae in the 20E-treated parasites compared to the control group at day 22, as might be expected there was a drop in the level of the pre-sausage stage larvae (Fig. 7a, b) at this time. There were no significant differences between the motility of parasites in the 20E-treated group and those in the control group, except that a slightly higher

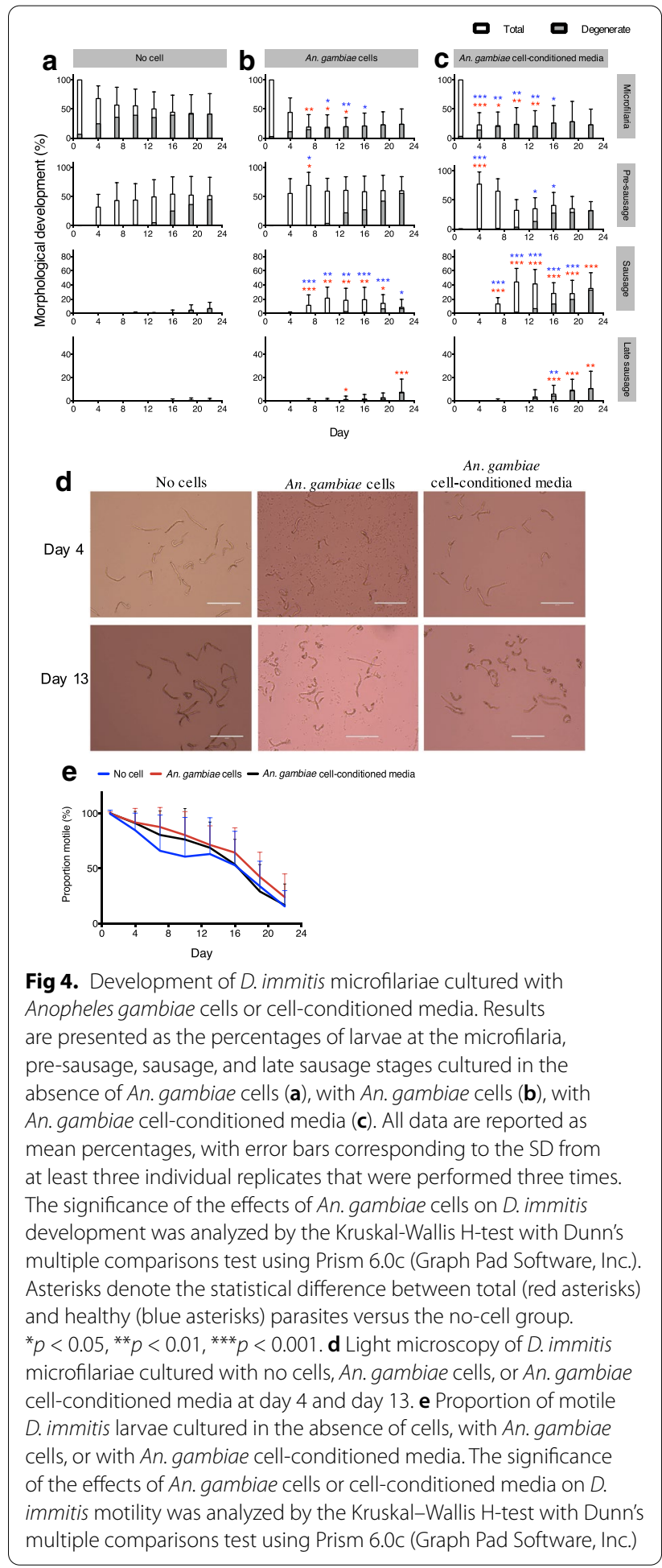

proportion were observed to be motile in the 20E-treated group on day 16 of incubation (Fig. 7c). 


\section{Discussion}

As there is very little publisher information on the in vitro development of $D$. immitis microfilariae [50-52], the aim of this research was to understand the developmental regulation underlying the development of $D$. immitis microfilariae in the hope of finding an alternative strategy to truncate the life cycle of the parasite.

\section{The composition of DMEM and Ham's F-12 culture media promote the in vitro development of $D$. immitis microfilariae}

In this study, D. immitis microfilariae developed into late L1 parasites that could be classified as pre-sausage, sausage, and late sausage stages. These late L1 stages differed considerably in their morphological appearances (Figs. 1, 2). However, similar to previous attempts to study the vector phase development of filarial nematodes [51-59], none of the D. immitis microfilariae in this study developed past the L1 stage, as no visible opening of the stoma was observed (Fig. 2) and no shedding of the cuticle took place between the transitions (Additional file 6: Video S5).

Among the commercially available culture media tested, DMEM and Ham's F-12 medium mixed at a 1:1 ratio provided a relatively favorable condition for the in vitro cultivation of microfilariae. In contrast, Schneider's insect medium and RPMI 1640 medium were both poor at sustaining parasite development and motility (Fig. 3). These findings are consistent with previous studies done by Sneller and Weinstein [50]. As motility changes with development, with microfilariae being more mobile than the more developed stages, the level of motility should not be taken as a measure of vitality. In our study, motility has been used as an indication of viability only, with motile larvae being assessed as alive and larvae that are immotile over a period of time being considered to be inactive and possibly dead. A closer look at the media composition showed that Schneider's insect medium contains high levels of intermediate compounds of the tricarboxylic acid (TCA) cycle while lacking in most $\mathrm{B}$ vitamins. As some filarial nematodes have low TCA cycle enzyme activity [60-62], it is possible that

$$
\begin{aligned}
& \text { Dim-nhr-7 5' - ATTGGTTCAATGAAGCAAG.................... ATGATGATT - 3' } \\
& \text { Dim-nhr-6 5' - CCGAACTCACTGAACCACT.................. ATGAATGAA - 3' } \\
& \text { Start codon }
\end{aligned}
$$

Fig 5. Putative ecdysone-response element (ECRE) in the promoter region upstream of the start codon of Dim-nhr-7 and Dim-nhr-6, downstream early regulatory genes of the ecdysone receptor (EcR). Upstream promoter sequence of Dim-nhr-7 nDi.2.2.2.g02617 and Dim-nhr-6 nDi.2.2.2.g04428 [49] were screened for EcRE. Start codons are marked with the blue box, putative EcRE are highlighted in yellow, and nucleotides that differ from the canonical EcRE are highlighted in red. intermediates of the TCA cycle do not contribute significantly to the overall energy metabolism of the parasite, while the lack of B vitamins may be detrimental to the growth and development of the microfilariae [63]. In addition, compared to DMEM and Ham's F-12, RPMI 1640 medium is comparatively lower in terms of content of a variety of inorganic salts and lacks sources of certain ions, such as copper, iron, and zinc $[64,65]$, which could have contributed to the poor growth and motility of the parasite. In comparison, the high levels of glucose, vitamins [63], and amino acids [66] in DMEM may be important energy sources for sustaining parasite motility, while the diversified components in Ham's F-12, including diamines [67], polyunsaturated fatty acid [68], organosulfur compound, purine, and pyrimidine [69], may contribute to the initiation of microfilaria development.

\section{Microfilaria development is driven by cues from the arthropod host cells but inhibited by mammalian-derived metabolites}

The importance of mosquito cells in microfilarial cultures has been reported $[52,57,70]$, but the secretion of microfilarial growth-promoting factors by mosquito cells has, to our knowledge, been demonstrated for the first time in this study. The favorable effect of mosquito cells was shown when $D$. immitis microfilariae were cultured with An. gambiae 4a-3B cells, where microfilariae that were cultured with mosquito cells showed early appearance of the sausage and late sausage stage parasites (Fig. 4). Because there was no observable interaction between the parasite and the cell layer underneath, we hypothesized that the growth-promoting factors of the cells may be secreted into the media. On further testing, media conditioned with $A n$. gambiae cells also had a similar effect, indicating that factors secreted by the mosquito cells provided conditions similar to the environment in the mosquito and promoted faster microfilarial development.

FBS was found to inhibit the development of the microfilariae in vitro (Fig. 3). A possible inhibitory component of the serum could be mammalian cholesterol derivatives, such as cholestenoic acid. Cholesterol derivatives have been found to contribute to the molting of D. immitis L3 larvae, a stage that develops inside the mammalian host [71]. This result indicates that the presence of mammalian cholesterol metabolites may imitate the mammalian host environment, in which microfilariae would be in a state of developmental arrest. However, it remains unclear why the motility of the parasites in the presence of FBS was reduced, nor is it clear whether the resultant change in motility reflects a change in viability or not, given observations, in vivo and in vitro, that motility changes naturally with development. 
a

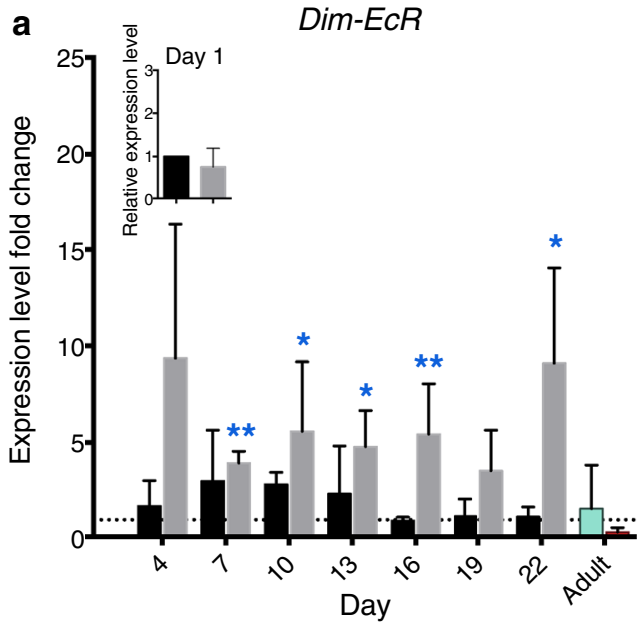

C

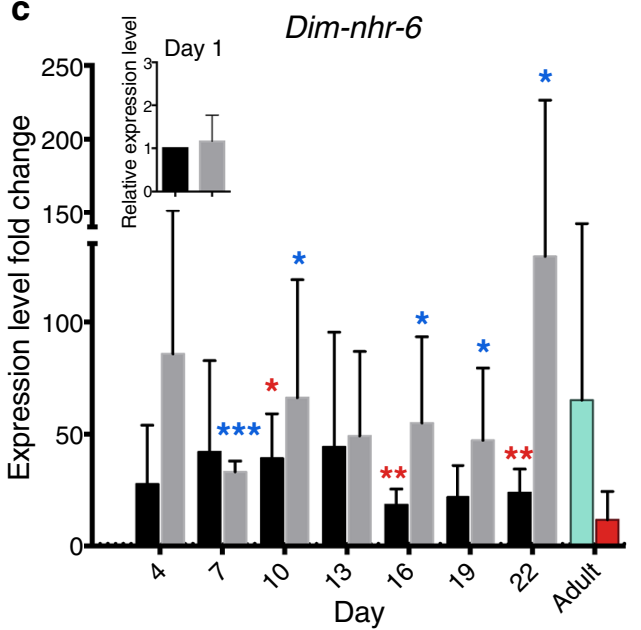

b

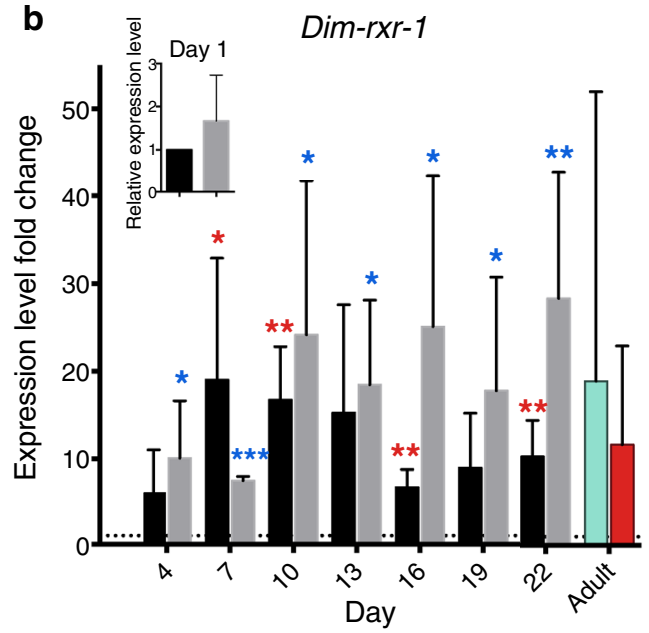

d

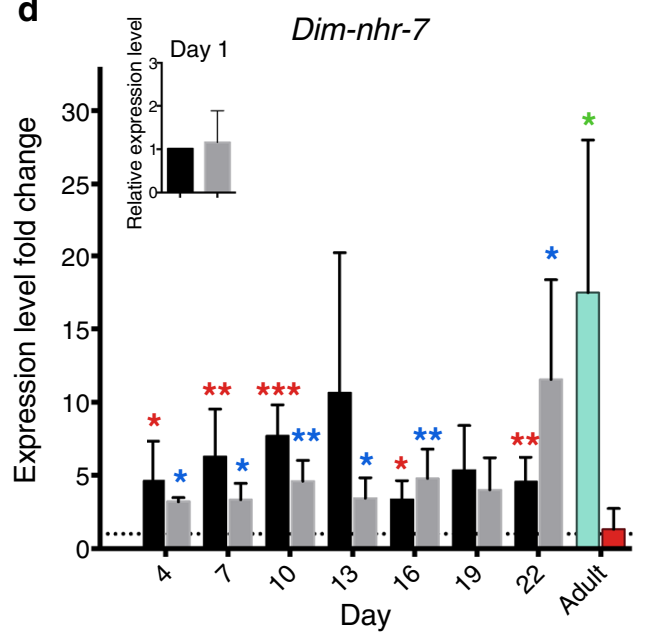

Fig 6. Transcript level of the ecdysone signaling system in D. immitis using droplet digital PCR. Fold changes for both no-cell and cell-conditioned media groups were calculated relative to day 1 of each medium. Fold changes for adults were calculated relative to day 1 of the cell-conditioned media group. Insert figures show transcript level at day 1 for both media. All transcript levels were normalized to three reference genes: Dim-GAPDH, Dim-Actin, and Dim- - -tubulin. a Dim-ECR, b Dim-rxr-1, c Dim-nhr-6, d Dim-nhr-7. All data are reported as mean fold change, with error bars corresponding to the SD from at least two individual replicates that were performed three times. The significance of the transcript level fold changes compared to day 1 baseline level was analyzed by the unpaired t-test with Welch's correction, two-tailed $p$ value, and $95 \%$ confidence interval using Prism 6.0c (Graph Pad Software, Inc.). Asterisks denote the statistical difference between no-cell microfilariae (red asterisks), cell-conditioned media microfilariae (blue asterisks), adults (green asterisks) vs day 1 groups (---). ${ }^{*} p<0.05,{ }^{* *} p<0.01,{ }^{* * *} p<0.001$

The upregulation of the ecdysone signaling pathway plays an important role in microfilaria developmental transitions Since the ecdysone signaling pathway plays a role in the morphological development of infective D. immitis L3 larvae, we hypothesized that the ecdysone signaling pathway may also play a role in the morphological changes observed in developing microfilaria culture. Sequence alignment of Dim-nhr-6 and Dim-nhr-7 with the EcRE consensus sequence identified putative EcRE in the promoter region of both genes, indicating that both could possibly act as downstream targets of the EcR (Fig. 5). Using ddPCR, we performed absolute quantification of the ecdysone signaling system gene transcripts and showed for the first time that morphologically distinct $D$. immitis L1 larvae displayed different transcript expression of the ecdysone signaling cascade. The results showed that the overall transcript expression of the ecdysone signaling system components increased gradually towards the 


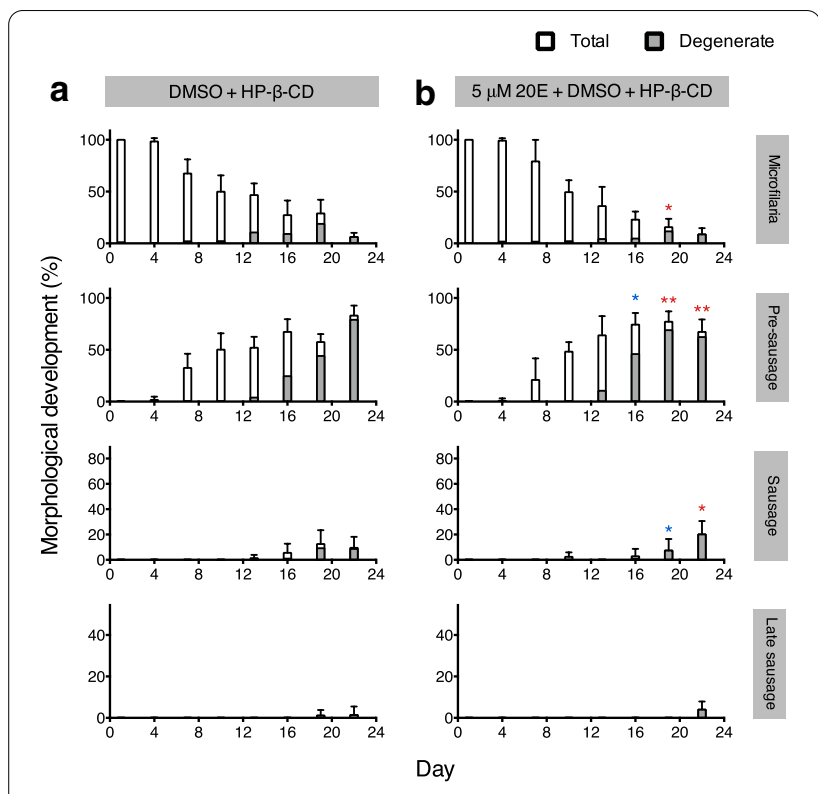

C $\quad$.. $\mathrm{DMSO}+\mathrm{HP}-\beta-\mathrm{CD}-5 \mu \mathrm{M} 2 \mathrm{OE}+\mathrm{DMSO}+\mathrm{HP}-\beta-\mathrm{CD}$

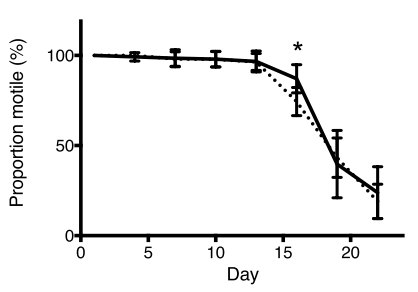

Fig 7. Development of $D$. immitis microfilariae treated with 20-hydroxyecdysone (HP- $\beta-C D)$. Results show percentages of larvae at the microfilaria, pre-sausage, sausage, and late sausage stages cultured in $0.05 \%$ DMSO + 0.004\% HP- $\beta-C D(\mathbf{a}), 5 \mu \mathrm{M}$ $20 \mathrm{E}+0.05 \% \mathrm{DMSO}+0.004 \% \mathrm{HP}-\beta-\mathrm{CD}(\mathbf{b})$. All data are reported as mean percentages, with error bars corresponding to the SD from at least three individual replicates. The significance of the effects of 20E on D. immitis development was analyzed by the Mann-Whitney U-test, two-tailed $P$ value, and 95\% confidence interval using Prism 6.0c (Graph Pad Software, Inc.). Asterisks denote the statistical difference between total (red asterisks) and healthy (blue asterisks) parasites vs DMSO + HP- $\beta$-CD group. ${ }^{*} p<0.05$, ${ }^{* *} p<0.01,{ }^{* * *} p<0.001$. c Proportion of motile D. immitis larvae cultured in $0.05 \% \mathrm{DMSO}+0.004 \% \mathrm{HP}-\beta-\mathrm{CD}$ or $5 \mu \mathrm{M} 20 \mathrm{E}+0.05 \%$ $\mathrm{DMSO}+0.004 \% \mathrm{HP}-\beta-\mathrm{CD}$. The significance of the effects of $20 \mathrm{E}$ on $D$. immitis the proportion of larvae that were motile was analyzed by the Mann-Whitney U-test, two-tailed $P$ value, and $95 \%$ confidence interval using Prism 6.0c (Graph Pad Software, Inc.). Asterisks denote the statistical difference between $5 \mu \mathrm{M} 20 \mathrm{E}+\mathrm{DMSO}+\mathrm{HP}-\beta-\mathrm{CD}$ vs DMSO + HP- $\beta-C D$ group. ${ }^{*} p<0.05,{ }^{* *} p<0.01,{ }^{* * *} p<0.001$

end of the 22-day in vitro incubation period in both the no-cell and An. gambiae cell-conditioning group, with the An. gambiae cell-conditioning group showing higher transcript levels for Dim-EcR, Dim-rxr-1, and Dim-nhr-6 genes (Fig. 6). Although microfilariae cultured without cells showed a higher transcript level of Dim-nhr-7 during the beginning of the culture period, the transcript level of microfilariae cultured with An. gambiae cell-conditioned medium rose quickly by day 22 . The finding that the overall transcript levels for the ecdysone signaling system components tended to be higher in microfilariae cultured in $A n$. gambiae cell-conditioned media, which corresponded to higher numbers of developing larval stages in the in vitro culture, suggests that factors secreted by An. gambiae cell may activate the EcR and the downstream ecdysone signaling system, and that the activation of the EcR may play an important role in the morphological transition of the microfilarial stage. It would be of interest, in a future study, to attempt to identify (a) factor(s) released by the insect cells which activate the EcR.

Interestingly, while all four genes of the ecdysone signaling cascade targeted in this study displayed a similar pattern of expression over time, the large increase in the overall transcript level of Dim-nhr-6 in developing larvae suggests that the EcR/RXR complex possibly upregulates the mRNA expression level of Dim-nhr-6 to a much higher extent than Dim-nhr-7, and that Dim-nhr-6 might be a crucial downstream target of EcR.

Given the increase in expression of genes in the ecdysone signaling pathway, it was interesting that 20 E significantly increased the numbers of $D$. immitis microfilariae that developed to later stages (Fig. 7). The incubations with $20 \mathrm{E}$ were conducted in the absence of the insect cells or their secretions in order to establish whether 20E itself had an effect. While 20E did increase the number of larvae developed, $20 \mathrm{E}$ by itself did not have as dramatic an effect on the development as did the factors released by, or changed by, the mosquito cells.

Although previously published results have mentioned the sex-specific pattern of the ecdysone cascade members in $D$. immitis adults was possibly due to gravidity of the female adult worms [37-40], ddPCR results from the present study showed that expression of the ecdysone cascade members could still be detected in adult male parasites, although the mRNA levels in males were lower than those of the females (Fig. 6). This result could mean that expression of the ecdysone signaling pathway in adult females is not entirely involved in microfilaria production and that further experiments are needed to determine the role of the ecdysone signaling pathway in the female and male adult stages.

\section{Conclusions}

To our knowledge, this study is the first to depict, in vitro, the detailed morphological transitions of D. immitis L1 larvae that occur inside the arthropod host. The present work shows in vitro culture conditions that allow microfilariae to develop to pre-sausage, sausage and late sausage stage L1 larvae. However, many of the later stage larvae were somewhat degenerated, development did not 
proceed through the L1/L2 molt, and the time it took for the development observed in vitro was longer than the time it takes in the mosquito. More work is needed to improve further the in vitro culture conditions to better mimic development in the mosquito, and perhaps the in vitro culture conditions will need to change as development occurs. Our results also demonstrate that factors secreted from mosquito cells play an important role in the development of $D$. immitis microfilariae and that the ecdysone cascade may contribute to the morphological changes seen with cultured microfilariae. These findings contribute to a better understanding of the developmental regulation of $D$. immitis microfilariae and open up new avenues for future investigations of the effects of ecdysone compounds and components released by mosquito cells on microfilarial development.

\section{Supplementary information}

Supplementary information accompanies this paper at https://doi. org/10.1186/s13071-020-04480-w.

Additional file 1: Table S1. Primer sequences used to amplify D. immitis ecdysone signaling cascade components.

Additional file 2: Video S1. Movement of $D$. immitis microfilaria.

Additional file 3: Video S2. Movement of D. immitis pre-sausage.

Additional file 4: Video S3. Movement of D. immitis sausage.

Additional file 5: Video S4. Movement of D. immitis late sausage.

Additional file 6: Video S5. Imaging of live larva. Microfilariae were cultured with An. gambiae 4a-3B cells and imaged over time to observe if any shedding took place. Pre-sausage stage starts from 00:00:00; sausage stage starts from 00:00:16; late sausage stage starts from 00:00:21.

\section{Abbreviations}

20E: 20-Hydroxyecdysone; ddPCR: Droplet digital PCR; ECR: Ecdysone receptor; EcRE: Ecdysone-response element; HP- $\beta$-CD: (2-Hydroxypropyl)- $\beta$ cyclodextrin; L1: First-stage (larvae); L3: Third-stage (larvae); ML: Macrocyclic lactone; $n h r$ : Nuclear hormone receptor genes; NR: Nuclear receptor; RXR: Mammalian retinoid $X$ receptor protein; USP: Ultraspiracle protein.

\section{Acknowledgements}

The following reagent was provided by the NIH/NIAID Filariasis Research Reagent Resource Center for distribution through BEI Resources, NIAID, NIH: Dirofilaria immitis, Strain Missouri, Microfilariae in Dog Blood (Live), NR-48907. We thank Zoetis Inc. for providing the female and male adult D. immitis (Kentucky strain). We also thank Dr. George K. Christophides for providing the Anopheles gambiae 4a-3B cell line, MRA-919, through BEl Resources. We would also like to thank Kathy Keller for her assistance with the microfilaria extractions, Dr. Thavy Long for her suggestions and input with the study design, Dr. Youssef Chebli for his assistance with the electron microscopy, Dr. Fernando Lopes for lending us the $\operatorname{EVOS}^{\mathrm{TM}} \mathrm{XL}$ inverted imaging system, and Dr. Petra Rohrbach for lending us the IncuCyte ZOOM system.

\section{Authors' contributions}

TCSK and RKP conceptualized and designed the experiments. TCSK carried out the experiments and the analysis. TCSK wrote the manuscript with input from RKP. Both authors read and approved the final manuscript.

\section{Funding}

The research leading to these results has received funding from a grant (No. RGPIN/2777-2012) from the Natural Sciences and Engineering Research
Council of Canada to RKP. This study was also supported by the Robert P. Harpur Fellowship in Parasitology at McGill University (TCSK), and the Graduate Excellence Fellowship at McGill University (TCSK). The funders did not play a role in the design of the study, collection, analysis and interpretation of data as well as the writing of the manuscript.

\section{Availability of data and materials}

The data supporting the conclusions of this article are included within the article.

\section{Ethics approval and consent to participate}

All experimental procedures were approved by McGill University in accordance with relevant guidelines and regulations.

\section{Consent for publication}

Not applicable.

\section{Competing interests}

The authors declare that they have no competing interests.

Received: 4 September 2020 Accepted: 7 November 2020

Published online: 09 December 2020

\section{References}

1. American Veterinary Medical Association. https://www.avma.org/. Accessed 12 Aug 2020.

2. Hampshire VA. Evaluation of efficacy of heartworm preventive products at the FDA. Vet Parasitol. 2005;133:191-5.

3. Blagburn BL, Dillon AR, Arther RG, Butler JM, Newton JC. Comparative efficacy of four commercially available heartworm preventive products against the MP3 laboratory strain of Dirofilaria immitis. Vet Parasitol. 2011;176:189-94.

4. Bourguinat C, Keller K, Blagburn B, Schenker R, Geary TG, Prichard RK. Correlation between loss of efficacy of macrocyclic lactone heartworm anthelmintics and P-glycoprotein genotype. Vet Parasitol. 2011;176:374-81.

5. Bourguinat C, Keller K, Bhan A, Peregrine A, Geary T, Prichard R. Macrocyclic lactone resistance in Dirofilaria immitis. Vet Parasitol. 2011;181:388-92.

6. Pulaski CN, Malone JB, Bourguinat C, Prichard R, Geary T, Ward D, et al. Establishment of macrocyclic lactone resistant Dirofilaria immitis isolates in experimentally infected laboratory dogs. Parasites Vectors. 2014;7:494.

7. Bourguinat $C$, Lee $A C Y$, Lizundia R, Blagburn BL, Liotta JL, Kraus MS, et al. Macrocyclic lactone resistance in Dirofilaria immitis: failure of heartworm preventives and investigation of genetic markers for resistance. Vet Parasitol. 2015;210:167-78.

8. Bourguinat C, Keller K, Xia J, Lepage P, McTier TL, Woods DJ, et al. Genetic profiles of ten Dirofilaria immitis isolates susceptible or resistant to macrocyclic lactone heartworm preventives. Parasites Vectors. 2017;10:504.

9. Centers for Disease Control and Prevention. https://www.cdc.gov/. Accessed 12 Aug 2020.

10. Loker E, Hofkin B. Parasitology: a conceptual approach. Boca Raton: CRC Press; 2015.

11. Underwood PC, Harwood PD. Survival and location of the microfilariae of Dirofilaria immitis in the dog. J Parasitol. 1939;25:23-33.

12. Ferreira CAC, de Pinho MV, Novo MTLM, Calado MMP, Gonçalves LAP, Belo SMD, et al. First molecular identification of mosquito vectors of Dirofilaria immitis in continental Portugal. Parasites Vectors. 2015;8:139.

13. Bowman DD, Atkins CE. Heartworm biology, treatment, and control. Vet Clin N Am Small Anim Pract. 2009;39:P1127-58.

14. Silaghi C, Beck R, Capelli G, Montarsi F, Mathis A. Development of Dirofilaria immitis and Dirofilaria repens in Aedes japonicus and Aedes geniculatus. Parasites Vectors. 2017;10:94.

15. Ledesma N, Harrington L. Fine-scale temperature fluctuation and modulation of Dirofilaria immitis larval development in Aedes aegypti. Vet Parasitol. 2015;209:93-100.

16. Montarsi F, Ciocchetta S, Devine G, Ravagnan S, Mutinelli F, Frangipane di Regalbono A, et al. Development of Dirofilaria immitis within the 
mosquito Aedes (Finlaya) koreicus, a new invasive species for Europe. Parasites Vectors. 2015:8:177.

17. Christensen BM, Hollander AL. Effect of temperature on vector-parasite relationships of Aedes trivittatus and Dirofilaria immitis. Proc Helminthol Soc Wash. 1978:45:115-9.

18. Taylor AE. The development of Dirofilaria immitis in the mosquito Aedes aegypti. J Helminthol. 1960;34:27-38.

19. Kartman L. Factors influencing infection of the mosquito with Dirofilaria immitis (Leidy, 1856). Exp Parasitol. 1953;2:27-78.

20. Aguinaldo AM, Turbeville JM, Linford LS, Rivera MC, Garey JR, Raff RA, et al. Evidence for a clade of nematodes, arthropods and other moulting animals. Nature. 1997;387:489-93.

21. Boulan L, Milán M, Léopold P. The systemic control of growth. Cold Spring Harb Perspect Biol. 2015;7:a019117.

22. Song Y, Villeneuve DL, Toyota K, Iguchi T, Tollefsen KE. Ecdysone receptor agonism leading to lethal molting disruption in arthropods: review and adverse outcome pathway development. Environ Sci Technol. 2017;51:4142-57.

23. Yamanaka N, Rewitz KF, O'Connor MB. Ecdysone control of developmental transitions: lessons from Drosophila research. Annu Rev Entomol. 2013;58:497-516.

24. Rewitz KF, Rybczynski R, Warren JT, Gilbert LI. The Halloween genes code for cytochrome P450 enzymes mediating synthesis of the insect moulting hormone. Biochem Soc Trans. 2006;34:1256-60.

25. Petryk A, Warren JT, Marques G, Jarcho MP, Gilbert LI, Kahler J, et al. Shade is the Drosophila P450 enzyme that mediates the hydroxylation of ecdysone to the steroid insect molting hormone 20-hydroxyecdysone. Proc Natl Acad Sci USA. 2003;100:13773-8.

26. Rewitz KF, Rybczynski R, Warren JT, Gilbert LI. Developmental expression of Manduca shade, the P450 mediating the final step in molting hormone synthesis. Mol Cell Endocrinol. 2006;247:166-74.

27. Schubiger M, Truman JW. The RXR ortholog USP suppresses early metamorphic processes in Drosophila in the absence of ecdysteroids. Development. 2000;127:1151.

28. Yao TP, Forman BM, Jiang Z, Cherbas L, Chen JD, McKeown M, et al. Functional ecdysone receptor is the product of ECR and Ultraspiracle genes. Nature. 1993:366:476-9.

29. Koelle MR, Talbot WS, Segraves WA, Bender MT, Cherbas P, Hogness DS. The Drosophila ECR gene encodes an ecdysone receptor, a new member of the steroid receptor superfamily. Cell. 1991;67:59-77.

30. Cherbas L, Lee K, Cherbas P. Identification of ecdysone response elements by analysis of the Drosophila Eip28/29 gene. Genes Dev. 1991;5:120-31.

31. Antoniewski C, Laval M, Lepesant J-A. Structural features critical to the activity of an ecdysone receptor binding site. Insect Biochem Mol Biol. 1993;23:105-14

32. Ashburner M. Sequential gene activation by ecdysone in polytene chromosomes of Drosophila melanogaster: I. Dependence upon ecdysone concentration. Dev Biol. 1973:35:47-61.

33. Ashburner M, Richards $G$. Sequential gene activation by ecdysone in polytene chromosomes of Drosophila melanogaster: III. Consequences of ecdysone withdrawal. Dev Biol. 1976;54:241-55.

34. Schumann I, Kenny N, Hui J, Hering L, Mayer G. Halloween genes in panarthropods and the evolution of the early moulting pathway in Ecdysozoa. R Soc Open Sci. 2018;5:180888.

35. Mendis AH, Rose ME, Rees HH, Goodwin TW. Ecdysteroids in adults of the nematode, Dirofilaria immitis. Mol Biochem Parasitol. 1983;9:209-26.

36. Mercer JG, Barker GC, Howells RE, Rees HH. Analysis of ecdysteroids in Onchocerca gibsoni, O. volvulus and nodule tissues. Trop Med Parasitol. 1989:40:434-9.

37. Shea C, Richer J, Tzertzinis G, Maina CV. An EcR homolog from the filarial parasite, Dirofilaria immitis requires a ligand-activated partner for transactivation. Mol Biochem Parasitol. 2010;171:55-63.

38. Shea C, Hough D, Xiao J, Tzertzinis G, Maina CV. An rxr/usp homolog from the parasitic nematode, Dirofilariaimmitis. Gene. 2004;324:171-82.

39. Crossgrove K, Laudet V, Maina CV. Dirofilaria immitis encodes Di-nhr-7, a putative orthologue of the Drosophila ecdysone-regulated E78 gene. Mol Biochem Parasitol. 2002;119:169-77.
40. Crossgrove K, Maina CV, Robinson-Rechavi M, Lochner MC. Orthologues of the Drosophila melanogaster E75 molting control gene in the filarial parasites Brugia malayi and Dirofilaria immitis. Mol Biochem Parasitol. 2008;157:92-7.

41. Tzertzinis G, Egaña AL, Palli SR, Robinson-Rechavi M, Gissendanner CR, Liu $C$, et al. Molecular evidence for a functional ecdysone signaling system in Brugia malayi. PLoS Negl Trop Dis. 2010;4:e625.

42. Warbrick EV, Barker GC, Rees HH, Howells RE. The effect of invertebrate hormones and potential hormone inhibitors on the third larval moult of the filarial nematode, Dirofilaria immitis, in vitro. Parasitology. 1993;107(Pt 4):459-63.

43. Fleming MW. Ascaris suum: role of ecdysteroids in molting. Exp Parasitol. 1985;60:207-10.

44. Dennis RD. Insect morphogenetic hormones and developmental mechanisms in the nematode, Nematospiroides dubius. Comp Biochem Physiol A Mol Integr Physiol. 1976;53:53-6.

45. Michalski ML, Griffiths KG, Williams SA, Kaplan RM, Moorhead AR. The $\mathrm{NIH}-\mathrm{NIAID}$ filariasis research reagent resource center. PLoS Negl Trop Dis. 2011;5:e1261.

46. Filariasis Research Reagent Resource Center (FR3). https://www.filariasis center.org/. Accessed 12 Aug 2020.

47. Quan P-L, Sauzade M, Brouzes E. dPCR: a technology review. Sensors (Basel). 2018;18:1271.

48. Lok JB. Signaling in parasitic nematodes: physicochemical communication between host and parasite and endogenous molecular transduction pathways governing worm development and survival. Curr Clin Microbiol Rep. 2016;3:186-97.

49. WormBase ParaSite. https://parasite.wormbase.org/. Accessed 01 Jan 2020.

50. Sneller V-P, Weinstein PP. In vitro development of Dirofilaria immitis microfilariae: Selection of culture media and serum levels. Int J Parasitol. 1982;12:233-8

51. Sawyer TK, Weinstein P. The in vitro development of microfilariae of the dog heartworm Dirofilaria immitis to the "sausage-form." J Parasitol. 1963a;49:218-24

52. Devaney E, Howells RE. Culture systems for the maintenance and development of microfilariae. Ann Trop Med Parasitol. 1979;73:139-44.

53. Sawyer TK, Weinstein PP. Studies on the microfilariae of the dog heartworm Dirofilaria immitis: separation of parasites from whole blood. J Parasitol. 1963:49:39-45.

54. Taylor AER. Maintenance of filarial worms in vitro. Exp Parasitol. 1960:9:113-20.

55. Sawyer TK, Weinstein PP. Survival of Dirofilaria immitis microfilariae in modified physiological saline solutions. J Parasitol. 1961;47:24.

56. Weinstein PP. Development in vitro of the microfilariae of Wuchereria bancrofti and of Litomosoides carinii as far as the sausage form. Trans $\mathrm{R}$ Soc Trop Med Hyg. 1963;57:236.

57. Devaney E, Howells RE. The development of exsheathed microfilariae of Brugia pahangi and Brugia malayi in mosquito cell lines. Ann Trop Med Parasitol. 1979;73:387-92.

58. Klein JB, Bradley RE. Induction of morphological changes in microfilariae from Dirofilaria immitis by in vitro culture techniques. J Parasitol. 1974:60:649-749.

59. Litchfield TM, Mackenzie CD, Varma MGR. The conditions required for the maintenance of Onchocerca lienalis microfilariae in vitro. Acta Trop. 1991;48:293-304.

60. Boreham PFL, Atwell RB. Dirofilariasis. Boca Raton: CRC Press; 1988.

61. Simón F, Siles-Lucas M, Morchón R, González-Miguel J, Mellado I, Carretón $E$, et al. Human and animal dirofilariasis: the emergence of a zoonotic mosaic. Clin Microbiol Rev. 2012;25:507-44.

62. Hochachka PW, Lutz PL, SickTJ, Rosenthal M. Surviving hypoxia: mechanisms of control and adaptation. Boca Raton: CRC Press; 1993.

63. Crompton DWT. Parasitology: human nutrition and parasitic infection. Cambridge: Cambridge University Press; 1993.

64. Shi HN, Scott ME, Koski KG, Boulay M, Stevenson MM. Energy restriction and severe zinc deficiency influence growth, survival and reproduction of Heligmosomoides polygyrus (Nematoda) during primary and challenge infections in mice. Parasitology. 1995;110(Pt 5):599-609. 
65. Briggs GM, Cheng AC, Lu NC. A study of mineral requirements in Caenorhabditis elegans. Nematologica. 1983;29:425-34.

66. Bird AF, Bird J. The structure of nematodes. Cambridge: Academic Press; 1991.

67. Wittich R-M, Kilian H-D, Walter RD. Polyamine metabolism in filarial worms. Mol Biochem Parasitol. 1987;24:155-62.

68. Mondal M, Kundu JK, Misra KK. Variation in lipid and fatty acid uptake among nematode and cestode parasites and their host, domestic fowl: host-parasite interaction. J Parasit Dis. 2016:40:1494-518.
69. Lee DL. The biology of nematodes. Boca Raton: CRC Press; 2010

70. Wood DE, Suitor EC Jr. In vitro development of microfilariae of Macacanema formosana in mosquito cell cultures. Nature. 1966;211:868-70.

71. Long T, Alberich M, André F, Menez C, Prichard RK, Lespine A. The development of the dog heartworm is highly sensitive to sterols which activate the orthologue of the nuclear receptor DAF-12. Sci Rep. 2020;10:11207.

\section{Publisher's Note}

Springer Nature remains neutral with regard to jurisdictional claims in published maps and institutional affiliations. 